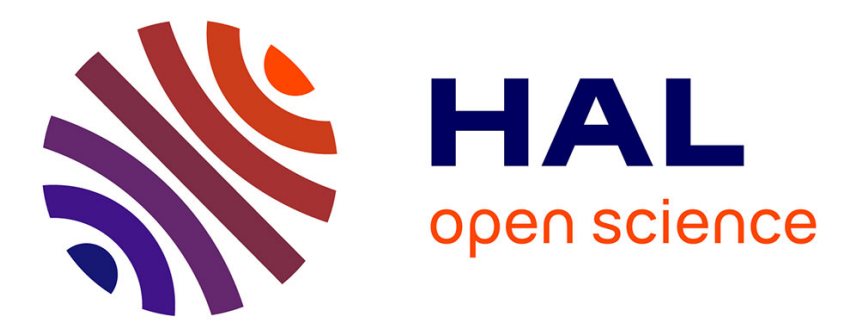

\title{
Pre-stimulus theta power is correlated with variation of motor evoked potential latency: a single-pulse TMS study
}

Zafer İşcan, Aaron Schurger, Marine Vernet, Jacobo Sitt, Antoni L Valero-Cabré

\section{To cite this version:}

Zafer İşcan, Aaron Schurger, Marine Vernet, Jacobo Sitt, Antoni L Valero-Cabré. Pre-stimulus theta power is correlated with variation of motor evoked potential latency: a single-pulse TMS study. Experimental Brain Research, 2018, 236 (11), pp.3003-3014. 10.1007/s00221-018-5359-3 . hal-02415205

\section{HAL Id: hal-02415205 \\ https://hal.science/hal-02415205}

Submitted on 17 Dec 2019

HAL is a multi-disciplinary open access archive for the deposit and dissemination of scientific research documents, whether they are published or not. The documents may come from teaching and research institutions in France or abroad, or from public or private research centers.
L'archive ouverte pluridisciplinaire HAL, est destinée au dépôt et à la diffusion de documents scientifiques de niveau recherche, publiés ou non, émanant des établissements d'enseignement et de recherche français ou étrangers, des laboratoires publics ou privés. 


\section{Experimental Brain Research}

\section{Pre-stimulus theta power predicts variation of motor evoked potential latency: A single- pulse TMS study \\ --Manuscript Draft--}

\begin{tabular}{|c|c|c|}
\hline \multicolumn{3}{|l|}{ Manuscript Number: } \\
\hline Full Title: & \multicolumn{2}{|c|}{$\begin{array}{l}\text { Pre-stimulus theta power predicts variation of motor evoked potential latency: A single- } \\
\text { pulse TMS study }\end{array}$} \\
\hline Article Type: & \multicolumn{2}{|l|}{ Research Article } \\
\hline \multirow[t]{5}{*}{ Funding Information: } & $\begin{array}{l}\text { Fondation pour la Recherche Médicale } \\
\text { (FRM) }\end{array}$ & Dr Marine Vernet \\
\hline & IHU-A-ICM-Translationnel & Dr Antoni Valero-Cabré \\
\hline & $\begin{array}{l}\text { Agence National de la Recherche (ANR), } \\
\text { projet Générique OSCILOSCOPUS }\end{array}$ & Dr Antoni Valero-Cabré \\
\hline & eraNET-JTC-HBP CAUSALTOMICS & Dr Antoni Valero-Cabré \\
\hline & $\begin{array}{l}\text { European Research Council } \\
\text { (640626) }\end{array}$ & Dr Aaron Schurger \\
\hline Abstract: & \multicolumn{2}{|c|}{$\begin{array}{l}\text { There has been a growing interest in the role of pre-stimulus oscillations on cortical } \\
\text { excitability in visual and motor systems. Prior studies focused on the relationship } \\
\text { between pre-stimulus neuronal activity and TMS-evoked motor evoked potentials } \\
\text { (MEPs) have reported heterogeneous results. We aimed to assess the role of pre- } \\
\text { stimulus neural activity on the latency of MEPs, which might enhance our } \\
\text { understanding of the variability of MEP signals, and potentially provide information on } \\
\text { the role played by cortical activity fluctuations in the excitability of cortico-spinal } \\
\text { pathways. Near-threshold single-pulse TMS (spTMS) was applied at random intervals } \\
\text { over the primary motor cortex of } 14 \text { healthy participants while they sat passively, in } \\
\text { order to trigger hand muscle contractions. Multichannel EEG was recorded during } \\
\text { spTMS blocks. Spearman correlations between both the variation in MEP onset } \\
\text { latencies and peak-to-peak MEP amplitudes, and the pre-stimulus power of EEG } \\
\text { oscillations were calculated across participants. We found that the variation in MEP } \\
\text { latency was positively correlated with pre-stimulus power in the theta range }(4-7 \mathrm{~Hz} \text { ) in } \\
\text { a broad time window (-3.1 } \mathrm{s}--1.9 \mathrm{~s} \text { ) preceding the spTMS generating the MEP. No } \\
\text { correlation between pre-stimulus power in any frequency band and MEP amplitude } \\
\text { was found. Our results show that pre-stimulus theta oscillations can be predictive of the } \\
\text { variation in MEP latency, an outcome measure determined by fiber conduction velocity } \\
\text { and synaptic delays along the corticospinal tract. This finding could prove useful for } \\
\text { clinicians using MEP latency-based information in pre- or intra-operative diagnostics of } \\
\text { corticospinal impairment. }\end{array}$} \\
\hline Corresponding Author: & \multicolumn{2}{|l|}{$\begin{array}{l}\text { Zafer Iscan, Ph.D. } \\
\text { INSERM CEA Cognitive Neuroimaging Unit } \\
\text { Gif-sur-Yvette, Île-de-France FRANCE }\end{array}$} \\
\hline \multicolumn{3}{|l|}{$\begin{array}{l}\text { Corresponding Author Secondary } \\
\text { Information: }\end{array}$} \\
\hline Corresponding Author's Institution: & \multicolumn{2}{|l|}{ INSERM CEA Cognitive Neuroimaging Unit } \\
\hline \multicolumn{3}{|l|}{$\begin{array}{l}\text { Corresponding Author's Secondary } \\
\text { Institution: }\end{array}$} \\
\hline First Author: & \multicolumn{2}{|l|}{ Zafer Iscan, Ph.D. } \\
\hline \multicolumn{3}{|l|}{ First Author Secondary Information: } \\
\hline \multirow[t]{4}{*}{ Order of Authors: } & \multicolumn{2}{|l|}{ Zafer Iscan, Ph.D. } \\
\hline & \multicolumn{2}{|l|}{ Aaron Schurger } \\
\hline & \multicolumn{2}{|l|}{ Marine Vernet } \\
\hline & \multicolumn{2}{|l|}{ Jacobo D Sitt } \\
\hline
\end{tabular}


Antoni Valero-Cabré

Order of Authors Secondary Information:

Author Comments:

Suggested Reviewers:

Vincenzo Romei

vromei@essex.ac.uk

Traian POPA

traianpopa@yahoo.com

Josep Valls-Solé

jvalls@clinic.ub.es

Ignasi Cos

ignasi.cos@gmail.com

Risto IImoniemi

risto.ilmoniemi@aalto.fi 
Pre-stimulus theta power predicts variation of motor evoked potential latency:

\section{A single-pulse TMS study}

Theta power predicts variation of MEP latency

Zafer İşcan ${ }^{1 *}$, Aaron Schurger ${ }^{1,2}$, Marine Vernet ${ }^{3}$, Jacobo D. Sitt ${ }^{1,2,3}$, Antoni Valero-Cabré ${ }^{3,4,5^{*}}$

${ }^{1}$ Cognitive Neuroimaging Unit, CEA DRF/Joliot Institute, INSERM, Université Paris-Sud, Université Paris-Saclay, NeuroSpin center, 91191 Gif-sur-Yvette - France

${ }^{2}$ Commissariat à l'Energie Atomique, Direction des Sciences du Vivant, I2BM, NeuroSpin center, 91191 Gif-sur-

Yvette - France

${ }^{3}$ Cerebral Dynamics, Plasticity and Rehabilitaion Group, Frontlab Team, CNRS UMR 7225, Institut du Cerveau et de la Moelle Epinière (ICM), Paris, France;

${ }^{4}$ Department of Anatomy and Neurobiology, Laboratory of Cerebral Dynamics, Boston University School of Medicine, Boston, MA

${ }^{5}$ Cognitive Neuroscience and Information Technology Research Program, Open University of Catalonia (UOC), Barcelona, Spain.

*Corresponding authors:

Dr. Zafer İşcan PhD, Post-doctoral researcher, Cognitive Neuroimaging Unit INSERM U992 / NeuroSpin / CEA-Saclay Bât 145, Point courier 156, 91191 Gif-sur-Yvette cedex, France. Phone: +33 782488508 E-mail: zaferiscan@yahoo.com ORCID ID : 0000-0001-9832-6591

Dr. Antoni Valero-Cabré, MD PhD, Groupe de Dynamiques Cérébrales, Plasticité et Rééducation. Frontlab, Institut du Cerveau et la Moelle Epinière, CNRS UMR 7225. 47 Boulevard de l'Hôpital, 75013 Paris, France. Phone: +33 1425727 41 63. E-mail: / avalerocabre@gmail.com

ORCID ID : 0000-0002-5033-2279 


\begin{abstract}
There has been a growing interest in the role of pre-stimulus oscillations on cortical excitability in visual and motor systems. Prior studies focused on the relationship between pre-stimulus neuronal activity and TMS-evoked motor evoked potentials (MEPs) have reported heterogeneous results. We aimed to assess the role of pre-stimulus neural activity on the latency of MEPs, which might enhance our understanding of the variability of MEP signals, and potentially provide information on the role played by cortical activity fluctuations in the excitability of cortico-spinal pathways. Nearthreshold single-pulse TMS (spTMS) was applied at random intervals over the primary motor cortex of 14 healthy participants while they sat passively, in order to trigger hand muscle contractions. Multichannel EEG was recorded during spTMS blocks. Spearman correlations between both the variation in MEP onset latencies and peak-to-peak MEP amplitudes, and the pre-stimulus power of EEG oscillations were calculated across participants. We found that the variation in MEP latency was positively correlated with pre-stimulus power in the theta range (4-7 $\mathrm{Hz})$ in a broad time window (-3.1 s $--1.9 \mathrm{~s})$ preceding the spTMS generating the MEP. No correlation between pre-stimulus power in any frequency band and MEP amplitude was found. Our results show that pre-stimulus theta oscillations can be predictive of the variation in MEP latency, an outcome measure determined by fiber conduction velocity and synaptic delays along the corticospinal tract. This finding could prove useful for clinicians using MEP latency-based information in pre- or intraoperative diagnostics of corticospinal impairment.
\end{abstract}

Keywords: Brain stimulation, pre-target onset, ongoing activity, latency variability, single-pulse TMS, motor evoked potentials, electroencephalography. 


\section{INTRODUCTION}

Fluctuations in the power of brain oscillations at different frequency bands have been shown to reflect cyclic modulations of cortical excitability, affecting the outcomes of visual detection (Thut et al. 2006; Busch et al. 2009; Romei et al. 2010; Busch and VanRullen 2010), discrimination (van Dijk et al. 2008), and perception in general (Romei et al. 2008a, b; Dugué et al. 2011; Jaegle and Ro 2014; Tomassini et al. 2017). In motor systems, particularly during movement preparation, event related synchronization/desynchronization in the alpha and beta bands has also been reported consistently in the literature (Serrien et al. 2003; Deiber et al. 2012; van Wijk et al. 2012; Zaepffel et al. 2013; Kajihara et al. 2015). In this context, methods that probe the state of cortical excitability and its temporal dynamics preceding the onset of a stimulus (either a visual target or a descending corticospinal volley) could contribute to our understanding of these phenomena.

In motor systems, the delivery of single-pulse transcranial magnetic stimulation (spTMS) to the primary motor cortex induces motor evoked potentials (MEPs) in peripheral muscles and is widely used to probe the integrity of descending corticospinal tract fibers. spTMS can also be used to estimate the level of corticospinal excitability (Kujirai et al. 1993; Nakamura et al. 1997; Chen et al. 1998; Sanger et al. 2001; Chen 2004) while quantifying the impact of motor training or the level of impairment due to brain lesions or disease. More sophisticated versions of these same approaches have also served to probe the causal implication of local frequency-specific brain rhythms in motor control processes. For example, spTMS to the primary motor cortex preceded by pulses at different intervals to prefrontal regions during a Go/No-go task has served to reveal a causal role for beta $(20 \mathrm{~Hz})$ rhythms in the 'top-down' control of inhibitory signals modulating MEP amplitudes (Picazio et al. 2018). Similarly, in the visual/attentional domain, the ability of occipital spTMS to elicit reportable visual phosphenes (Elkin-Frankston et al. 2011) has been applied to reveal alpha (10 Hz) excitability fluctuations (Dugué et al. 2011) in primary visual areas and demonstrate periodic attentional sampling of visual stimuli according to ongoing theta (5 Hz) cycles (Dugue et al. 2015).

One disadvantage of MEPs to evaluate the physiological mechanisms subtending primary motor and corticospinal function is the large trial-to-trial variability in the amplitude of these motor evoked responses (Kiers et al. 1993; Wassermann 2002; Saisanen et al. 2008). Previous studies that tried to associate this variability with ongoing oscillatory brain activity have reported heterogeneous results (Zarkowski et al. 2006; Sauseng et al. 2009; Mäki and Ilmoniemi 2010; Berger et al. 2014; Iscan et al. 2016). Zarkowski et al. (Zarkowski et al. 2006) showed that MEP amplitude correlated negatively with pre-TMS stimulus power in the high alpha $(10-13 \mathrm{~Hz})$ frequency band and positively with power in the low gamma $(30-60 \mathrm{~Hz})$ band. The ratio of power in the low gamma to high alpha band during the pre-pulse period was the best predictor of MEP amplitude. Sauseng et al. (Sauseng et al. 2009) confirmed this relationship between power and 
MEP amplitude for the alpha band only. However other studies failed to replicate these findings (Iscan et al. 2016). For

example, Maki and Ilmoniemi (Mäki and Ilmoniemi 2010) found no correlation at the trial-by-trial level. However, on average, the amplitude of spontaneous oscillations in the low beta $(15-18 \mathrm{~Hz})$ range measured over the stimulated primary motor cortex (M1) (but not over other non-stimulated areas) was weaker just before large versus small amplitude MEPs. Berger et al. (Berger et al. 2014) found no correlation between pre-stimulus (-500 ms to 0 ms) EEG amplitude of oscillations in various frequency bands and MEP amplitude across trials.

Compared to MEP amplitude, which has been shown to have high intra-individual and inter-individual variability, MEP latency is a much more stable measure within individuals (Kiers et al. 1993; Cacchio et al. 2009, 2011), with the potential to provide information on signal conduction processes operating at the cortical and subcortical level (Kallioniemi et al. 2015). In spite of its stability, there are considerably fewer papers on MEP latency compared to MEP amplitude. Latency is highly influenced by conduction properties and synaptic delays at central (cerebral and spinal) and peripheral divisions of the nervous system (Fernández et al. 2013). Bergmann et al. (Bergmann et al. 2012) triggered spTMS over M1 during non-REM sleep according to the phase of spontaneous (> 1 Hz) neocortical slow oscillations (SO) to show that MEPs were larger and their latencies were shorter during SO up-states than during down-states, which revealed that MEP latency can be modulated by brain states.

Hamada et al. (Hamada et al. 2013) showed that inter-individual variability in the offline effects (also referred to as after-effects following the end of a repetitive TMS pattern) generated by continuous theta burst stimulation (cTBS) over M1 could be predicted by the latency of MEPs recorded before stimulation. This result was also replicated by Huang and Mouraux (Huang and Mouraux 2015). Nonetheless, clinically, no interhemispheric differences in MEP latency have been found in healthy participants (Saisanen et al. 2008) or in brain tumor patients (Picht et al. 2012). Indeed, no effect of clinical variables on MEP latency has been found when such are corrected for the subjects' height (determining the distance that cortico-spinal volleys need to complete from M1 to reach the monitored peripheral muscles).

Observations in healthy participants found large inter-subject differences in MEP latency. Nonetheless, it should be noted that Huang and Mouraux (Huang and Mouraux 2015) did not find a significant correlation between latency and height. Regarding age, Saisanen et al. (Saisanen et al. 2008) reported an effect of age on resting motor threshold (RMT), but Picht et al. (Picht et al. 2012) were unable to corroborate this effect (possibly due to the young age range of the studied cohort). Recently, latency variability between subjects was analyzed during TMS motor mapping in a large cohort of neurosurgical patients. Authors identified, for the three muscles they analyzed, gender and antiepileptic drug intake as muscle-specific factors influencing MEP latency together with resting motor threshold, tumor side and tumor location (Sollmann et al. 2017). 
In the current study, we used spTMS to test the role of pre-stimulus cortical fluctuations on MEP latency. We

suspected that latency would give more consistent results since it is a more stable measure than the amplitude. Therefore we focused our analysis on MEP latency and checked pre-stimulus activity in different frequency bands to see which bands covary with this outcome measure. We also measured MEP amplitudes as in other studies for completeness.

Contributing a novel finding to prior research, we found that pre-stimulus power in the theta range $(4-7 \mathrm{~Hz}) \mathrm{was}$ positively correlated with the variation in MEP latencies in a pre-stimulus time window $(-3.1 \mathrm{~s}--1.9 \mathrm{~s})$. Our results show that the power of pre-stimulus theta oscillations can be predictive of the variation in MEP latencies.

\section{METHODS}

\section{Participants}

Fourteen right-handed young adults ( 3 males and 11 females) between 18-39 years of age (mean: $25 \pm 6, \mathrm{SD}$ ) participated in the study. They had no history of psychiatric or neurological conditions, they were not taking any medication known to affect motor cortical excitability at the time of the study and did not have any contraindications for TMS. All tolerated the procedure without any side effect or complication. They gave written informed consent to participate in the study, which followed international guidelines and recommendations for the safe use of TMS (Rossi et al. 2009). The study had been approved by the local ethics committee (CPP Ile de France 1) and was conducted in adherence with the Declaration of Helsinki.

\section{Material}

The experiment was controlled using Matlab (MathWorks, Natick MA, USA) and the Psychophysics Toolbox (Brainard 1997) running on a PC (operating system: Windows). Synchronization between the computer, TMS stimulator, EEG, and EMG systems was provided by a trigger generator (Master-8, AMPI, Jerusalem, Israel), USB, and coaxial connections.

TMS pulses were delivered using a biphasic repetitive magnetic stimulator (Super Rapid², Magstim, Withland, $\mathrm{UK}$ ) and a 70-mm diameter figure-of-eight coil. Individual anatomical MRI (T1-weighted TR = $2300 \mathrm{~ms}, \mathrm{TE}=4.18 \mathrm{~ms}$, matrix size $=256 \times 256$, and 176 sagittal slices with $1 \mathrm{~mm}$ thickness, acquired on a 3T Siemens scanner), when available, or template anatomical MRI, were fed to a neuronavigation system (Brainsight, Rogue Inc., Montreal, Canada). Neuronavigation was used at all times to ensure that once localized, the same M1 spot was consistently stimulated throughout the experiment. 
EEG was continuously acquired from 13 scalp contacts (10-20 EEG system locations F3, Fz, F4, FC1, FC2, C3,

$\mathrm{Cz}, \mathrm{C} 4, \mathrm{CP} 1, \mathrm{CP} 2, \mathrm{P} 3, \mathrm{Pz}, \mathrm{P} 4)$, using a TMS-compatible EEG system (BrainAmp DC and BrainVision Recording Software, Brain Products GmbH, Munich, Germany) and TMS-compatible electrodes. The reference and ground were placed on the nose and right earlobe contralateral to the stimulation site on electrode C3. Skin-electrode impedance was maintained at all times below $5 \mathrm{k} \Omega$. The signal was digitized at a sampling rate of $5 \mathrm{kHz}$.

EMG was acquired using surface electrodes attached to the skin in a belly tendon montage over the first dorsal interosseus (FDI) and the abductor pollicis brevis (APB) muscles in the right hand, contralateral to the cortical stimulation site in participants' dominant left primary motor cortex using a data acquisition system (PowerLab 26T (LTS) and Chart Software, ADInstruments, New Zealand). The signal was digitized at a sampling rate of $40 \mathrm{kHz}$, and then down-sampled offline to $5 \mathrm{kHz}$ prior to data analysis.

\section{Procedure}

Participants were seated in a comfortable chair with their elbows flexed at approximately $90^{\circ}$ and both hands resting on a table. M1 of the left dominant hemisphere was stimulated. The coil was consistently held tangentially to the scalp, with the handle pointing posterior with a $45^{\circ}$ angle from the sagittal plane of the head for optimal orientation (Brasil-Neto et al. 1992). In this orientation, induced electrical current travels in posterior to anterior direction (Janssen et al. 2015). Once the motor hot spot was identified, it was stimulated with consistent coil location, orientation and tilt throughout the rest of the experiment. The resting motor threshold (RMT) was determined as the lowest intensity capable of inducing 5 out of 10 MEPs of at least $50 \mu \mathrm{V}$ peak-to-peak amplitude (Rossini et al. 1994). For our group of 14 participants the average RMT was $70 \pm 5 \%$ of the maximum stimulator output (MSO).

Following the RMT determination procedure, 4 blocks of 30 single-pulses at $110 \%$ of RMT were delivered at random intervals of approximately 10 seconds drawn from a Poisson distribution (98\% of intervals in the range of 9 to 11 seconds), while EEG and EMG were recorded. A mean interval of 10 seconds was chosen in order to strike a balance between the following three considerations: (1) Allowing enough time for effects of the prior pulse to decay completely; (2) Leaving a "clean" $5 \mathrm{sec}$ window of time before each pulse; and (3) Maximizing the number of trials in the session. In three participants stimulation intensity was increased to $120 \%, 125 \%$ and $135 \%$ of RMT, respectively to ensure the presence of consistent MEPs.

The instruction given to participants was to relax, fixate a small cross on a computer display in front of them and avoid eye movements and eye blinks several seconds before and immediately after each TMS pulse. Blocks were separated by a $2-5$ min break. 


\section{Data Analysis and Statistics}

Data analyses and statistics were performed using Matlab (MathWorks, Inc) and the Statistics Toolbox for Matlab. Preprocessing and epoching of the EEG data were carried out using the FieldTrip toolbox for Matlab (Oostenveld et al. 2011). Epochs were extracted from 5 seconds before each trigger (the TMS pulse onset). Since analyses focused on the EEG time course before the TMS pulse was delivered, the artifact created by the TMS pulse on subsequent EEG data (i.e., affecting recordings in the time period immediately following the TMS pulse) were not a concern. No filtering was applied to the data during pre-processing. Trials with ocular artifacts during the final 3 seconds prior to the TMS pulse were rejected by visual inspection. EEG recordings and MEPs from trials on which the TMS coil was not well positioned (i.e. stimulation was delivered more than $5 \mathrm{~mm}$ away from our FDI pre-defined hotspot) were identified in the neuronavigation log file and excluded from subsequent data analyses. Trials with excessive noise due to TMS coil contact or friction on EEG contacts, muscle contraction or eye-blinks were removed from the analysis. Out of 120 trials, the average number of trials used in the analyses was $63 \pm 22$.

EMG data were first down-sampled by a factor of 8 , from $40 \mathrm{kHz}$ to $5 \mathrm{kHz}$, in order to match the sampling rate of the EEG data. EMG data epochs extended from $-0.5 \mathrm{sec}$ to $+0.5 \mathrm{sec}$ with respect to the TMS trigger pulse. The MEP is a very brief event occurring within the first $100 \mathrm{~ms}$ after the TMS pulse, but well after ( 20-25 ms) the very brief artifact in the EMG time series introduced by the TMS pulse (see Fig. 1).

For each epoch of EMG data, we subtracted the mean over the baseline interval from 8 to 18 ms after the TMS pulse, defined as the "quiet" interval in between the TMS artifact and the onset of the motor-evoked potential. This results in a very precise alignment of the amplitude of the signal at the time of MEP onset. We then computed the peak-to-peak MEP amplitude (P2P) and MEP onset latency, as follows:

$\mathrm{P} 2 \mathrm{P}$ was computed as the difference between the maximum and the minimum EMG amplitude from 20 to $35 \mathrm{~ms}$ after the TMS pulse. Latency was defined as the first time-point after the TMS pulse for which the amplitude exceeded $5 \%$ of the baseline-to-first peak amplitude (in absolute value) (Huang and Mouraux 2015). In Fig. 2, latency and MEP amplitude (P2P) distributions (zoomed in $0-1 \mathrm{mV}$ ) of subjects are presented using histogram plots.

In Fig. 3, single trial MEP responses of a representative participant (Subject \#5) are presented.

\section{Variability of MEP onset latencies}

Coefficient of quartile variation (CQV) (Bonett 2006) was used to measure the variability of MEP amplitudes and MEP onset latencies between participants. This is a more robust method than the coefficient of variation, as it is less 
sensitive to the deviations from normality (Bonett 2006). Briefly, it is the ratio of interquartile range (Q3 - Q1) to the sum of first (Q1) and third (Q3) quartiles in data:

$$
C Q V=\frac{Q_{3}-Q_{1}}{Q_{3}+Q_{1}}
$$

For details of the application of the method see (Iscan et al. 2016).

\section{Power of pre-stimulus EEG oscillations}

Pre-stimulus power between 4.5 to 0.5 seconds was estimated from the spectrum calculated with a Fast Fourier Transform (FFT), with a Hanning window to remove discontinuities in the edges of the signal. In the single-trial analysis the power was calculated separately for each pre-stimulus interval. For the group-level analysis, the power was averaged across all trials in each frequency band, electrode and participant. Frequencies of interest covered delta (1-3 Hz), theta (4$7 \mathrm{~Hz})$, alpha $(8-13 \mathrm{~Hz})$, beta $(14-30 \mathrm{~Hz})$ and gamma (31-100) bands.

\section{Correlations between Power of pre-stimulus oscillations and MEP amplitude}

We computed a Spearman correlation between pre-stimulus power and MEP amplitude or the CQV of MEP amplitude for delta $(1-3 \mathrm{~Hz})$, theta $(4-7 \mathrm{~Hz})$, alpha $(8-13 \mathrm{~Hz})$, beta $(14-30 \mathrm{~Hz})$ and gamma (31-100) bands. The time window for the calculation was between 4.5 to 0.5 seconds before the stimulus.

\section{Correlations between Power of pre-stimulus oscillations and variability of MEP onset latencies}

First, we inspected data for the presence of multivariate outliers. In our study we employed the commonly-used Mahalanobis distance (MD) method to detect outliers (Lepage et al. 2012; Ballard et al. 2014). One subject who had an MD value $(M D=37.75)$ bigger than the critical $\chi^{2}$ value of $36.12(\mathrm{df}=14 ; \alpha=0.001)$, was excluded from the analysis. Then, we computed a Spearman correlation between pre-stimulus power in the frequencies of interest and the CQV of MEP onset latencies. Significance was estimated using cluster-based permutation statistics (Maris and Oostenveld 2007). The neighborhood for the cluster was defined with the triangulation method based on a two-dimensional projection of the electrode position. The number of permutations was set to 10000 . 
We first tested the correlation on a large pre-stimulus window (between 4.5 to 0.5 seconds). Then we performed a post-hoc cluster-based permutation test to isolate more precisely the effect in time using time-frequency analysis in this window with a step size of 0.1 seconds.

\section{RESULTS}

\section{Correlation between pre-stimulus band power and the MEP amplitude across participants}

No correlation between power and MEP amplitudes across participants was found in any frequency sub-bands, at delta $(1-3 \mathrm{~Hz})$, theta $(4-7 \mathrm{~Hz})$, alpha $(8-13 \mathrm{~Hz})$, beta $(14-30 \mathrm{~Hz})$ and gamma (31-100) (see Methods). Moreover, no correlation of pre-stimulus power was found with the MEP amplitude variability (i.e. CQV of MEP amplitude).

\section{Correlation between pre-stimulus theta oscillations and CQV of MEP onset latencies}

Fig. 4 shows the topography of the Spearman correlation between pre-stimulus theta power and CQV of MEP onset latencies using cluster-based permutation statistics for all participants in our cohort $(\mathrm{N}=14)$ and in a subcohort of 13 subjects (without the participant identified as an outlier according to the Mahalanobis distance method). It can be observed that for both analyses in the precentral and parietal regions, theta power correlated positively with the variability (i.e. $\mathrm{CQV}$ ) of latencies.

In Fig. 5 correlations between power of pre-stimulus theta oscillations and latency of MEPs are provided with significant cluster channels in the $-3.1 \mathrm{~s}$ to $-1.9 \mathrm{~s}$ range using time-frequency analysis.

When the time-frequency analysis was performed for all subjects, the significant cluster $(\mathrm{p}=0.021)$ covered $-3.2 \mathrm{~s}$ to $-1.6 s$ (See Fig. 6).

\section{DISCUSSION}

Driven by a growing interest in the role of pre-stimulus oscillations on cortical excitability, the current study uses EEG recordings to explore potential associations between pre-stimulus rhythmic activity in different frequency bands and parameters of the MEPs generated by stimulating the primary motor cortex. We focused on testing the role of cortical pre-stimulus rhythmic neural activity on MEP amplitude and its latency, a less studied outcome measure which might help further elucidate the source of variability for motor-evoked signals, and inform on how this influences excitability across corticospinal pathways. 
We found that pre-TMS power in the theta range $(4-7 \mathrm{~Hz})$ was positively correlated with variation in latency of

MEPs across a broad time window (-3.1 s - -1.9 s) preceding the stimulation pulse. On the contrary, we did not find evidence for an association between power in any frequency band and MEP amplitude.

Fig. 2 shows the variation of peak-to-peak (P2P) amplitudes and latencies between subjects (see also Fig. 3 for MEP signals on representative subject, \#5). We tried to explain this variability by testing for a potential role of prestimulus power in different EEG frequency bands. Nonetheless, no significant correlation was found between pre-stimulus power and MEP amplitude. This result is in line with those reported by some previous studies (Mäki and Ilmoniemi 2010; Berger et al. 2014; Iscan et al. 2016) using similar approaches. In contrast, two previous studies (Zarkowski et al. 2006; Sauseng et al. 2009) did report a significant correlation, a discrepancy which could be explained by the low reliability of such studies based on a very small sample of participants ( $\mathrm{N}=4$ and $\mathrm{N}=6$, respectively).

The main novelty of the present study is the potential relationship between the power of pre-stimulus oscillations and MEP onset latencies during prolonged spTMS sessions. Our results (Figs. 4 and 5) reveal a significant correlation between pre-stimulus theta power and the CQV of latencies across subjects. The time window (i.e. $-3.1--1.9 \mathrm{~s}$ ) in which this correlation was found shows that the variation in latency depends on the pre-stimulus EEG up to several seconds before the TMS pulse. It should be noted that the duration of the pre-stimulus window showing significant correlations remained similar even when an outlier participant was included in the analysis.

Two main question are worth-discussing. First, our data reveal that power in the theta, but not beta (as these reflect motor-related oscillatory activity), alpha, or delta bands predicted variability in MEP latency. In direct support of this finding, a recent paper, showed that cortical excitability during long periods of wakefulness correlated significantly with oscillations in theta power, whereas the power of delta, alpha, or beta rhythms failed to predict the variability of MEP amplitude (Ly et al. 2016). Second, in our study, MEP latency but not MEP amplitude correlated significantly with theta rhythms. The selective impact of theta power on MEP latency variability, a parameter depending on conduction and spinal (and also neuromuscular) synaptic delays for corticospinal descending volleys could be explained by the higher stability of MEP latency measures compared to amplitude. Indeed, a recent report (Tomassini et al. 2017), showed that the phase of theta oscillations predicted perceptual performance more than one second before a movement. Although that study was based on theta phase rather than theta power and the predicted output was perceptual performance instead of MEP latency, it is relevant in terms of showing correlations in a pre-stimulus time range very far in advance of stimulus onset.

Our interpretations might be tempered by two potential methodological shortcomings. First, for practical reasons, our study recorded EEG from 13 scalp contacts. Second, in some participants the intensity of TMS output had to be adjusted to ensure that MEPs were evoked by stimulation, a fact that could have increased the variability and added noise to our data. The number of subjects that participated in our study $(\mathrm{N}=13)$ was relatively modest but within the range of 
several other studies looking for a relationship between oscillations and MEP measures ((Mäki and Ilmoniemi 2010),

$\mathrm{N}=14$; (Sauseng et al. 2009), N=6; (Iscan et al. 2016), N=17). A substantial proportion of the TMS trials collected with EEG had to be excluded from the analysis due to several types of artifacts. However, on average we still had more than twice the optimal number of trials (Goldsworthy et al. 2016).

To the best of our knowledge, this is the first study to find a correlation between pre-stimulus theta power and the latency of MEPs. The exploitation of this link could prove useful to clinicians using latency information for pre-operative diagnostics of patients with tumors (Sollmann et al. 2017) or as an indicator of lower extremity and gait impairment (Peters et al. 2017). It could also serve to better assess latency information by including the variation stemming from changes in theta power. Moreover, our results could contribute to the development of brain-state triggered stimulation (Walter et al. 2012; Gharabaghi et al. 2014), a promising therapeutic methodology (Gharabaghi et al. 2014; Zrenner et al. 2016; Karabanov et al. 2016) based on tailoring the delivery of TMS to motor cortical regions (in terms of intensity, timing/phase and/or frequency) based on ongoing patterns of cortical activity in order to maximize its modulatory impact on cortico-spinal systems (Romei et al. 2016).

\section{ACKNOWLEDGMENTS}

MV was supported by the Fondation pour la Recherche Médicale (FRM). AS was supported by an ERC Starting Grant (640626). AV-C group's research is supported by IHU-A-ICM-Translationnel, and Agence National de la Recherche (ANR), projet Générique OSCILOSCOPUS and eraNET-JTC-HBP CAUSALTOMICS. Authors would like to thank Julià L Amengual from Brain dynamics, Cognition and Rehabilitation Group, Frontlab Team, Institut du Cerveau et de la Moelle Epinière (ICM), Paris, France, for his valuable comments and suggestions on the manuscript.

\section{CONFLICT OF INTEREST}

The authors declare no conflict of interest.

\section{REFERENCES}

Ballard KJ, Savage S, Leyton CE, et al (2014) Logopenic and Nonfluent Variants of Primary Progressive Aphasia Are Differentiated by Acoustic Measures of Speech Production. PLoS One 9:e89864

Berger B, Minarik T, Liuzzi G, et al (2014) EEG oscillatory phase-dependent markers of corticospinal excitability in the resting brain. Biomed Res Int 2014:. doi: 10.1155/2014/936096 
Bergmann TO, Molle M, Schmidt MA, et al (2012) EEG-guided transcranial magnetic stimulation reveals rapid shifts

10.1523/JNEUROSCI.4792-11.2012

Bonett DG (2006) Confidence interval for a coefficient of quartile variation. Comput Stat Data Anal 50:2953-2957. doi: http://dx.doi.org/10.1016/j.csda.2005.05.007

Brainard DH (1997) The Psychophysics Toolbox. Spat Vis 10:433-436

Brasil-Neto JP, Cohen LG, Panizza M, et al (1992) Optimal focal transcranial magnetic activation of the human motor cortex: effects of coil orientation, shape of the induced current pulse, and stimulus intensity. J Clin Neurophysiol 9:132-136

Busch NA, Dubois J, VanRullen R (2009) The phase of ongoing EEG oscillations predicts visual perception. J Neurosci 29:7869-7876. doi: 10.1523/JNEUROSCI.0113-09.2009

Busch NA, VanRullen R (2010) Spontaneous EEG oscillations reveal periodic sampling of visual attention. Proc Natl Acad Sci U S A 107:16048-16053. doi: 10.1073/pnas.1004801107

Cacchio A, Cimini N, Alosi P, et al (2009) Reliability of transcranial magnetic stimulation-related measurements of tibialis anterior muscle in healthy subjects. Clin Neurophysiol 120:414-419. doi: https://doi.org/10.1016/j.clinph.2008.11.019

Cacchio A, Paoloni M, Cimini N, et al (2011) Reliability of TMS-related measures of tibialis anterior muscle in patients with chronic stroke and healthy subjects. J Neurol Sci 303:90-94. doi: https://doi.org/10.1016/j.jns.2011.01.004

Chen R (2004) Interactions between inhibitory and excitatory circuits in the human motor cortex. Exp Brain Res 154:110. doi: 10.1007/s00221-003-1684-1

Chen R, Tam A, Bütefisch C, et al (1998) Intracortical Inhibition and Facilitation in Different Representations of the Human Motor Cortex. J Neurophysiol 80:2870-2881

Deiber M-P, Sallard E, Ludwig C, et al (2012) EEG alpha activity reflects motor preparation rather than the mode of action selection. Front Integr Neurosci 6:59. doi: 10.3389/fnint.2012.00059

Dugue L, Marque P, VanRullen R (2015) Theta oscillations modulate attentional search performance periodically. J Cogn Neurosci 27:945-958. doi: 10.1162/jocn_a_00755

Dugué L, Marque P, VanRullen R (2011) The phase of ongoing oscillations mediates the causal relation between brain excitation and visual perception. J Neurosci 31:11889-93. doi: 10.1523/JNEUROSCI.1161-11.2011 
Elkin-Frankston S, Fried P, Rushmore RJ, Valero-Cabre A (2011) From qualia to quantia: a system to document and quantify phosphene percepts elicited by non-invasive neurostimulation of the human occipital cortex. J Neurosci Methods 198:149-157. doi: 10.1016/j.jneumeth.2011.02.013

Fernández V, Valls-Sole J, Relova JL, et al (2013) Recommendations for the clinical use of motor evoked potentials in multiple sclerosis. Neurol (English Ed 28:408-416. doi: https://doi.org/10.1016/j.nrleng.2013.09.003

Gharabaghi A, Kraus D, Leão MT, et al (2014) Coupling brain-machine interfaces with cortical stimulation for brainstate dependent stimulation: enhancing motor cortex excitability for neurorehabilitation. Front Hum Neurosci 8:122. doi: 10.3389/fnhum.2014.00122

Goldsworthy MR, Hordacre B, Ridding MC (2016) Minimum number of trials required for within- and between-session reliability of TMS measures of corticospinal excitability. Neuroscience 320:205-9. doi: 10.1016/j.neuroscience.2016.02.012

Hamada M, Murase N, Hasan A, et al (2013) The role of interneuron networks in driving human motor cortical plasticity. Cereb Cortex 23:1593-1605. doi: 10.1093/cercor/bhs147

Huang G, Mouraux A (2015) MEP Latencies Predict the Neuromodulatory Effect of cTBS Delivered to the Ipsilateral and Contralateral Sensorimotor Cortex. PLoS One 10:e133893

Iscan Z, Nazarova M, Fedele T, et al (2016) Pre-stimulus Alpha Oscillations and Inter-subject Variability of Motor Evoked Potentials in Single- and Paired-Pulse TMS Paradigms . Front. Hum. Neurosci. 10:504

Jaegle A, Ro T (2014) Direct control of visual perception with phase-specific modulation of posterior parietal cortex. J Cogn Neurosci 26:422-432. doi: 10.1162/jocn_a_00494

Janssen AM, Oostendorp TF, Stegeman DF (2015) The coil orientation dependency of the electric field induced by TMS for M1 and other brain areas. J Neuroeng Rehabil 12:47. doi: 10.1186/s12984-015-0036-2

Kajihara T, Anwar MN, Kawasaki M, et al (2015) Neural dynamics in motor preparation: From phase-mediated global computation to amplitude-mediated local computation. Neuroimage 118:445-455. doi: https://doi.org/10.1016/j.neuroimage.2015.05.032

Kallioniemi E, Pitkänen M, Säisänen L, Julkunen P (2015) Onset Latency of Motor Evoked Potentials in Motor Cortical Mapping with Neuronavigated Transcranial Magnetic Stimulation. Open Neurol J 9:62-69. doi: $10.2174 / 1874205 \times 01509010062$

Karabanov A, Thielscher A, Siebner HR (2016) Transcranial brain stimulation: closing the loop between brain and 
stimulation. Curr Opin Neurol. doi: 10.1097/WCO.0000000000000342

Kiers L, Cros D, Chiappa KH, Fang J (1993) Variability of motor potentials evoked by transcranial magnetic stimulation. Electroencephalogr Clin Neurophysiol 89:415-423

Kujirai T, Caramia MD, Rothwell JC, et al (1993) Corticocortical inhibition in human motor cortex. J Physiol 471:501519. doi: 10.1113/jphysiol.1993.sp019912

Lepage J-F, Morin-Moncet O, Beaulé V, et al (2012) Occlusion of LTP-Like Plasticity in Human Primary Motor Cortex by Action Observation. PLoS One 7:e38754

Ly JQM, Gaggioni G, Chellappa SL, et al (2016) Circadian regulation of human cortical excitability. 7:11828

Mäki H, Ilmoniemi RJ (2010) EEG oscillations and magnetically evoked motor potentials reflect motor system excitability in overlapping neuronal populations. Clin Neurophysiol 121:492-501. doi: 10.1016/j.clinph.2009.11.078

Maris E, Oostenveld R (2007) Nonparametric statistical testing of EEG-and MEG-data. J Neurosci Methods 164:177190

Nakamura H, Kitagawa H, Kawaguchi Y, Tsuji H (1997) Intracortical facilitation and inhibition after transcranial magnetic stimulation in conscious humans. J Physiol 498:817-823

Oostenveld R, Fries P, Maris E, Schoffelen J-M (2011) FieldTrip: Open Source Software for Advanced Analysis of MEG, EEG, and Invasive Electrophysiological Data. Intell Neurosci 2011:1:1--1:9. doi: 10.1155/2011/156869

Peters HT, Dunning K, Belagaje S, et al (2017) Navigated Transcranial Magnetic Stimulation: A Biologically Based Assay of Lower Extremity Impairment and Gait Velocity. Neural Plast 2017:6971206. doi: $10.1155 / 2017 / 6971206$

Picazio S, Veniero D, Ponzo V, et al (2018) Prefrontal Control over Motor Cortex Cycles at Beta Frequency during Movement Inhibition. Curr Biol 24:2940-2945. doi: 10.1016/j.cub.2014.10.043

Picht T, Strack V, Schulz J, et al (2012) Assessing the functional status of the motor system in brain tumor patients using transcranial magnetic stimulation. Acta Neurochir (Wien) 154:2075-2081. doi: 10.1007/s00701-012-1494-y

Romei V, Brodbeck V, Michel C, et al (2008a) Spontaneous fluctuations in posterior alpha-band EEG activity reflect variability in excitability of human visual areas. Cereb Cortex 18:2010-2018. doi: 10.1093/cercor/bhm229

Romei V, Gross J, Thut G (2010) On the role of prestimulus alpha rhythms over occipito-parietal areas in visual input regulation: correlation or causation? J Neurosci 30:8692-7. doi: 10.1523/JNEUROSCI.0160-10.2010 
Romei V, Rihs T, Brodbeck V, Thut G (2008b) Resting electroencephalogram alpha-power over posterior sites indexes baseline visual cortex excitability. Neuroreport 19:203-208. doi: 10.1097/WNR.0b013e3282f454c4

Romei V, Thut G, Silvanto J (2016) Information-Based Approaches of Noninvasive Transcranial Brain Stimulation. Trends Neurosci 39:782-795. doi: https://doi.org/10.1016/j.tins.2016.09.001

Rossi S, Hallett M, Rossini PM, Pascual-Leone A (2009) Safety, ethical considerations, and application guidelines for the use of transcranial magnetic stimulation in clinical practice and research. Clin Neurophysiol 120:2008-2039. doi: 10.1016/j.clinph.2009.08.016

Rossini PM, Barker AT, Berardelli A, et al (1994) Non-invasive electrical and magnetic stimulation of the brain, spinal cord and roots: basic principles and procedures for routine clinical application. Report of an IFCN committee. Electroencephalogr Clin Neurophysiol 91:79-92

Saisanen L, Julkunen P, Niskanen E, et al (2008) Motor potentials evoked by navigated transcranial magnetic stimulation in healthy subjects. J Clin Neurophysiol 25:367-372. doi: 10.1097/WNP.0b013e31818e7944

Sanger TD, Garg RR, Chen R (2001) Interactions between two different inhibitory systems in the human motor cortex. J Physiol 530:307-317. doi: 10.1111/j.1469-7793.2001.03071.x

Sauseng P, Klimesch W, Gerloff C, Hummel FC (2009) Spontaneous locally restricted EEG alpha activity determines cortical excitability in the motor cortex. Neuropsychologia 47:284-288. doi: http://dx.doi.org/10.1016/j.neuropsychologia.2008.07.021

Serrien DJ, Fisher RJ, Brown P (2003) Transient increases of synchronized neural activity during movement preparation: influence of cognitive constraints. Exp Brain Res 153:27-34. doi: 10.1007/s00221-003-1578-2

Sollmann N, Bulubas L, Tanigawa N, et al (2017) The variability of motor evoked potential latencies in neurosurgical motor mapping by preoperative navigated transcranial magnetic stimulation. BMC Neurosci 18:5. doi: $10.1186 / \mathrm{s} 12868-016-0321-4$

Thut G, Nietzel A, Brandt SA, Pascual-Leone A (2006) Alpha-band electroencephalographic activity over occipital cortex indexes visuospatial attention bias and predicts visual target detection. J Neurosci 26:9494-9502. doi: 10.1523/JNEUROSCI.0875-06.2006

Tomassini A, Ambrogioni L, Medendorp WP, Maris E (2017) Theta oscillations locked to intended actions rhythmically modulate perception. Elife 6:. doi: 10.7554/eLife.25618

van Dijk H, Schoffelen J-M, Oostenveld R, Jensen O (2008) Prestimulus Oscillatory Activity in the Alpha Band 
Predicts Visual Discrimination Ability. J Neurosci 28:1816 LP-1823

van Wijk BCM, Beek PJ, Daffertshofer A (2012) Neural synchrony within the motor system: what have we learned so far? Front Hum Neurosci 6:252. doi: 10.3389/fnhum.2012.00252

Walter A, Murguialday AR, Rosenstiel W, et al (2012) Coupling BCI and cortical stimulation for brain-state-dependent stimulation: Methods for spectral estimation in the presence of stimulation after-effects . Front. Neural Circuits 6

Wassermann EM (2002) Variation in the response to transcranial magnetic brain stimulation in the general population. Clin Neurophysiol 113:1165-1171

Zaepffel M, Trachel R, Kilavik BE, Brochier T (2013) Modulations of EEG Beta Power during Planning and Execution of Grasping Movements. PLoS One 8:e60060. doi: 10.1371/journal.pone.0060060

Zarkowski P, Shin CJ, Dang T, et al (2006) EEG and the Variance of Motor Evoked Potential Amplitude. Clin EEG Neurosci 37:247-251. doi: 10.1177/155005940603700316

Zrenner C, Belardinelli P, Müller-Dahlhaus F, Ziemann U (2016) Closed-Loop Neuroscience and Non-Invasive Brain Stimulation: A Tale of Two Loops. Front Cell Neurosci 10:92. doi: 10.3389/fncel.2016.00092 


\section{FIGURE CAPTIONS}

Fig. 1 An example of EMG record including a single TMS pulse (at time =0) and the corresponding Motor Evoked Potential (MEP). The two main parameters that are extracted to characterize the MEP, are the peak-to-peak (P2P) amplitude and the latency

Fig. 2 Latency (left) and MEP amplitude (P2P) (right) distributions of the 14 subjects (S1-S14) participating in the study. Colors represent the number of occurrences (trial number)

Fig. 3 Single trial MEP responses of a representative participant (Subject \#5) zoomed into 20 to 35 ms after TMS pulse

Fig. 4 Topography of the correlation between pre-stimulus (-4.5 to -0.5 s) theta oscillations and CQV of MEP onset latencies for the complete cohort of 14 subjects (left panel) and for a subset of 13 participants (right panel, dataset excluding the participant identified as an outlier). Significantly clustered channels are marked with bigger labels (N=14), one cluster (FC1, FC2, CP2, P3, Pz, P4) with $\mathrm{p}=0.019 ; \mathrm{N}=13$, two clusters (FC1 \& FC2 with p=0.048; P3 \& Pz with $\mathrm{p}=0.049)$

Fig. 5 Correlations between pre-stimulus (-3.1 s to -1.9 s) theta oscillations and CQV of MEP onset latencies. Significant cluster channels $(\mathrm{p}=0.038)$ are labelled with black dots. Analyses were performed on a cohort of N=13 (i.e., the participant identified as an outlier was excluded)

Fig. 6 Correlations between pre-stimulus (-3.2 s to -1.6 s) theta oscillations and CQV of MEP onset latencies. Significant cluster channels $(\mathrm{p}=0.021)$ are marked with black dots. $\mathrm{N}=14$ 


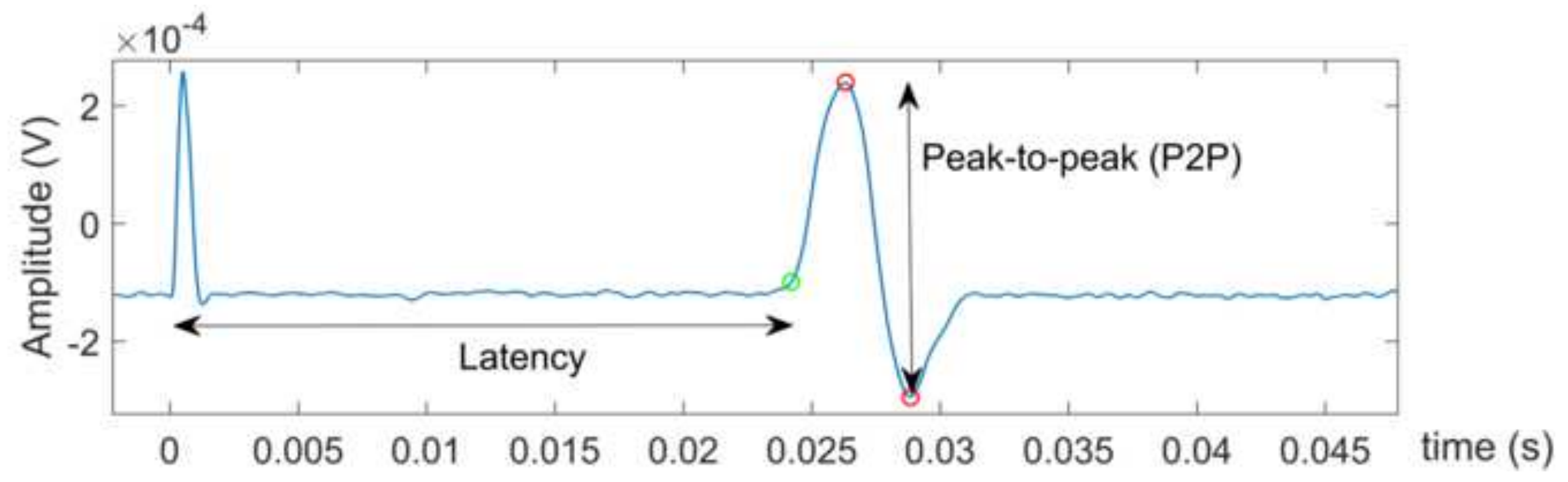



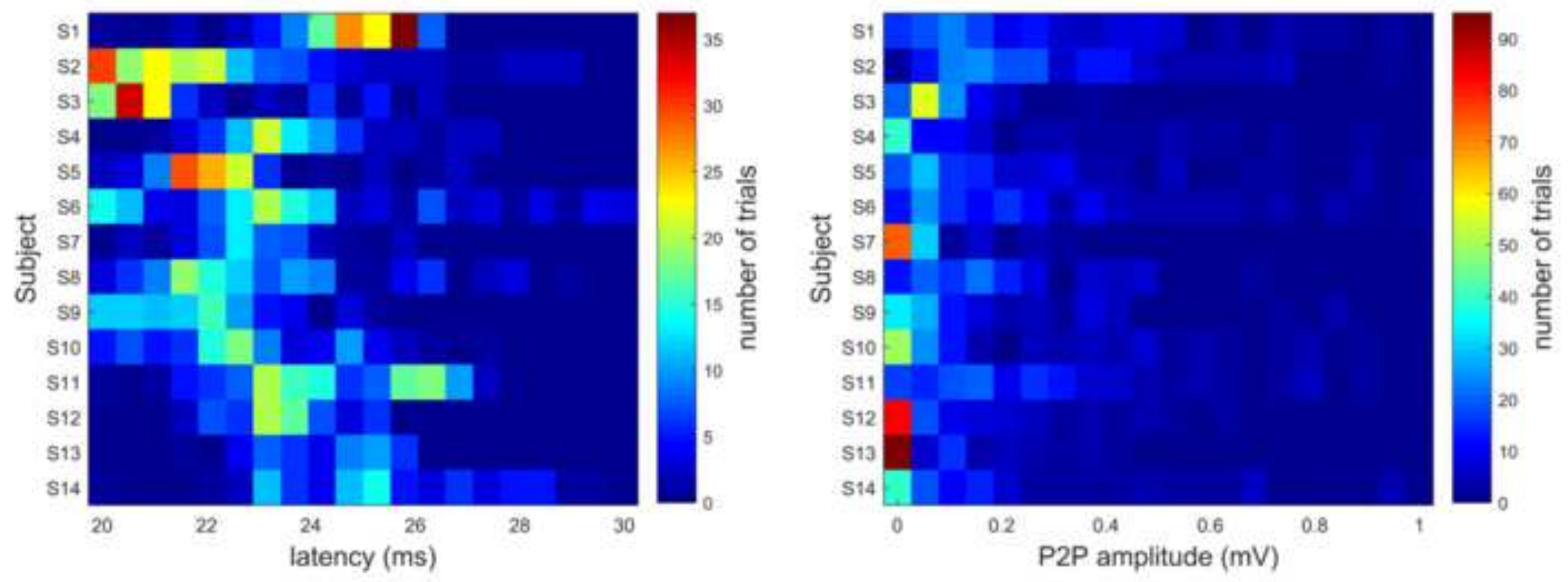

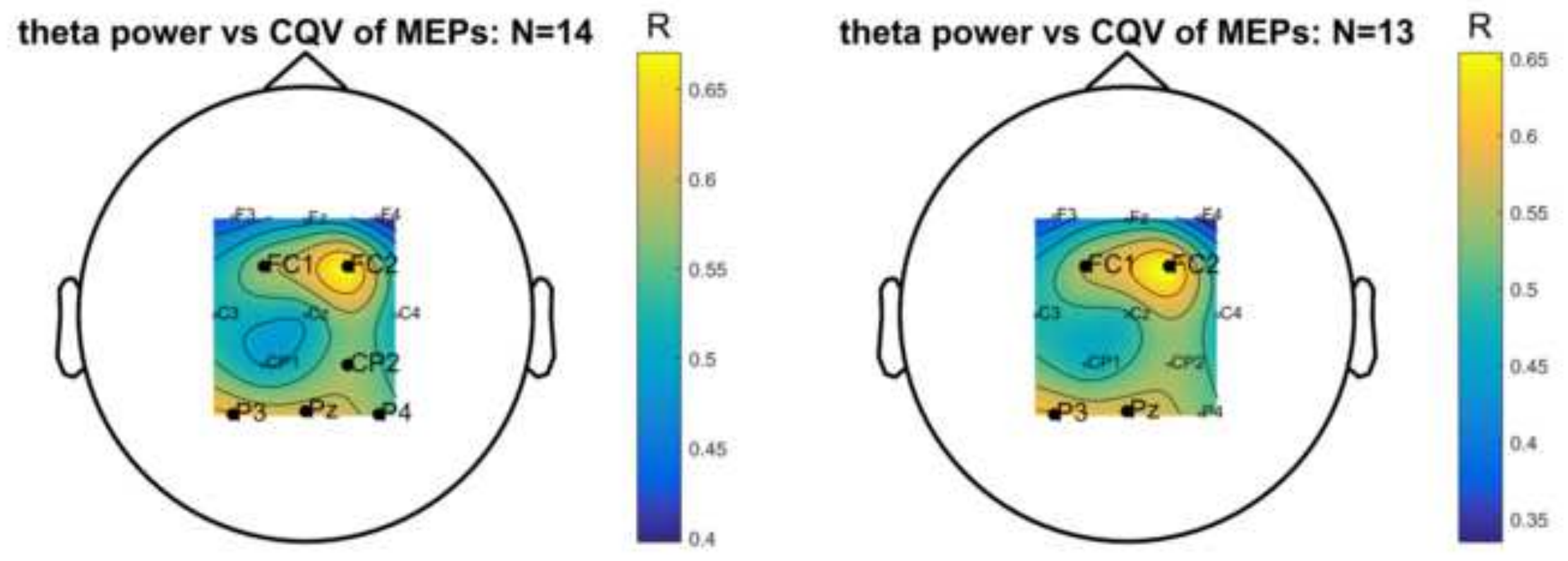

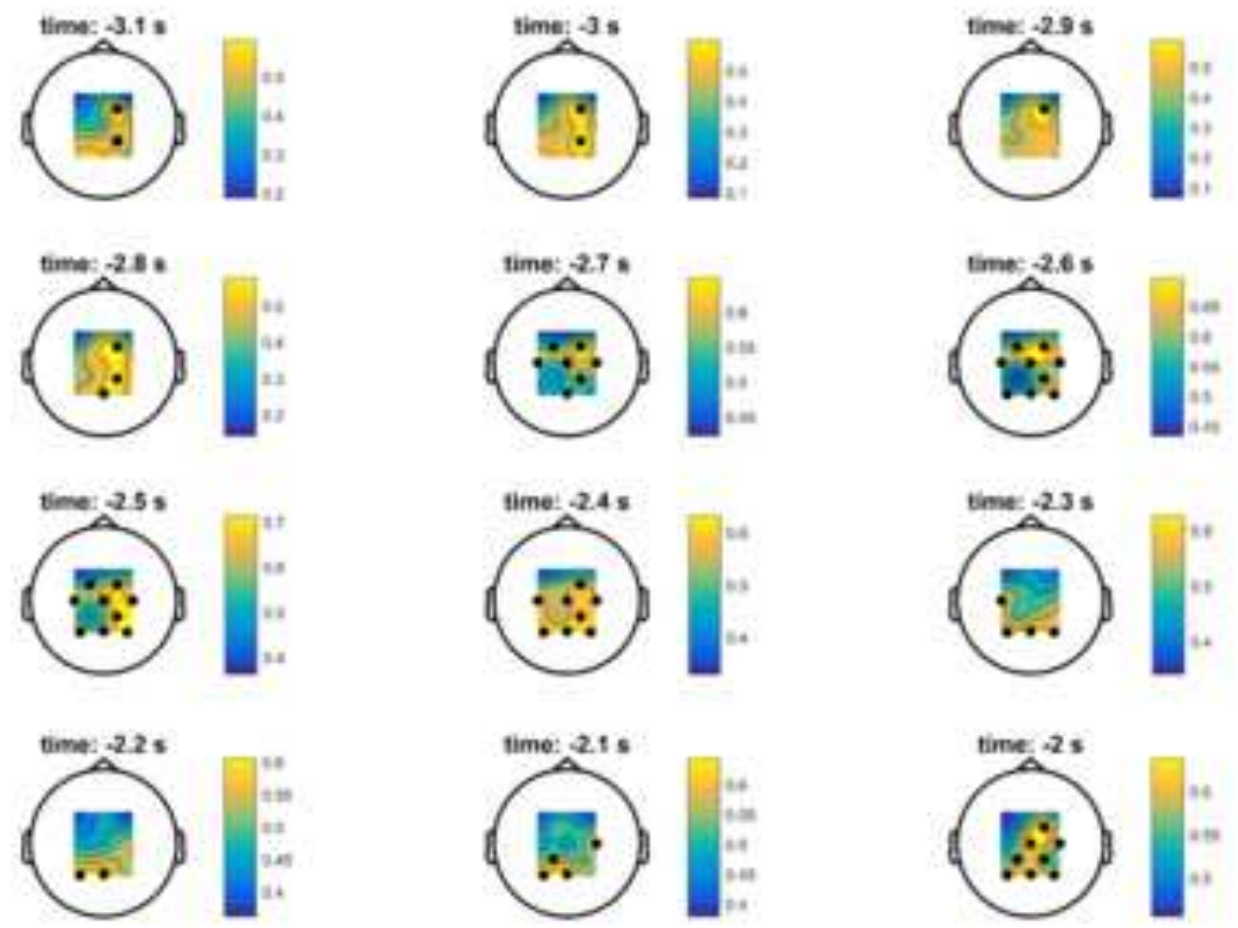

time:2.1s

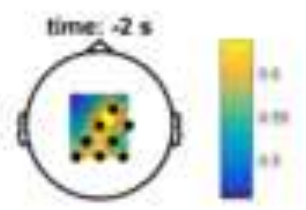

time:-1.2. 


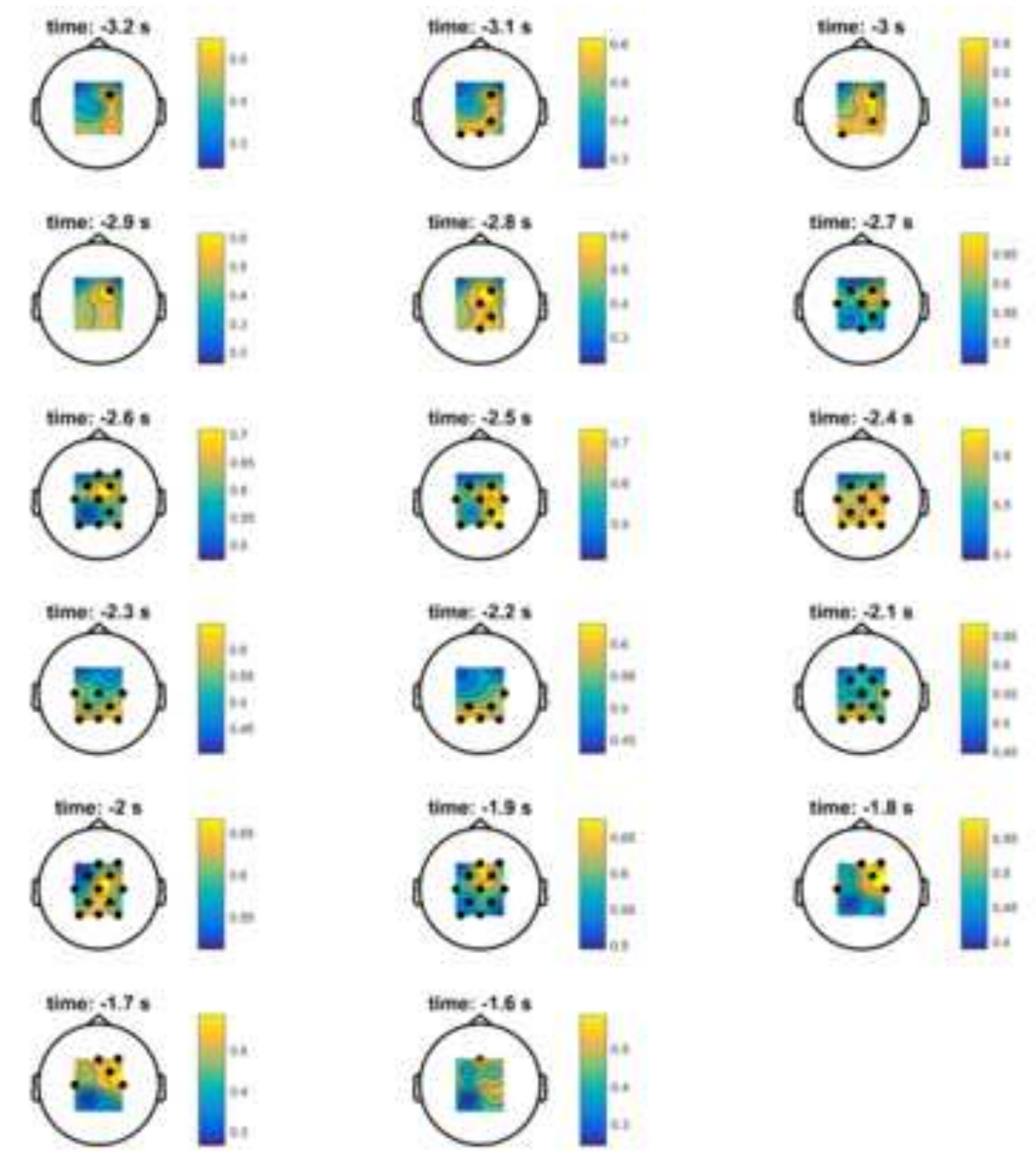

